\title{
Current State of Combined Kidney and Pancreas Transplantation
}

\author{
Anja Richter $^{\mathrm{a}}$ Susan Lerner ${ }^{\mathrm{b}}$ Bernd Schröppe $\mathrm{l}^{\mathrm{a}, \mathrm{b}}$ \\ ${ }^{a}$ Renal Division and ${ }^{b}$ Recanati/Miller Transplantation Institute, Mount Sinai School of Medicine, New York, N.Y., USA
}

\section{Key Words}

Pancreas transplantation - Diabetes mellitus •

Complications of pancreas transplantation -

Glycemic control · Graft survival

\begin{abstract}
Glycemic control via the use of exogenous insulin injections in diabetic patients is incomplete, resulting in multiple longterm complications, such as retinopathy, neuropathy, vasculopathy, and nephropathy. The goal of whole-pancreas and kidney transplantation is to achieve long-term insulin independence and correct uremia. The proposed benefits of pancreas and kidney transplantation are improved quality of life, prevention of recurrent diabetic nephropathy, freedom from exogenous insulin, stabilization or improvement in secondary complications, and improved mortality. No other regimen of insulin delivery or renal replacement besides pancreas and kidney transplantation can achieve this level of physiologic regulation.

Copyright $\odot 2011$ S. Karger AG, Basel
\end{abstract}

\section{Introduction}

The world's first clinical pancreas transplant was performed, simultaneously with a kidney graft, in 1966 at the University of Minnesota. The frequency of pancreas transplantation has increased as the results have improved such that over 22,000 pancreas transplantations have now been performed in the US, with almost 1,400 transplants in 2006 in the US [1]. The purpose of the review is to summarize the types of pancreas transplants, timing of transplantation, its impact on patient survival and preexisting diabetic complications, and surgical as well as immunosuppressant drug complications.

\section{Types of Pancreas Transplantation}

Pancreas transplants are done in three different categories of diabetic recipients, classified according to their kidney function. $72 \%$ of pancreas transplants are performed as simultaneous pancreas-kidney transplants (SPK). Since 1996, there has been a relative increase in pancreas after kidney transplantation (PAK) and pancreas transplants alone (PTA) but these numbers started to decline in 2004.

\section{KARGER}

Fax +4161306 1234 E-Mail karger@karger.ch www.karger.com
Bernd Schröppel, MD

Mount Sinai Medical Center

One Gustave L. Levy Place Box 1104

New York, NY 10029-6574 (USA)

Tel. +1 212659 8003, Fax +1 212241 2138, E-Mail bernd.schroppel@mountsinai.org 


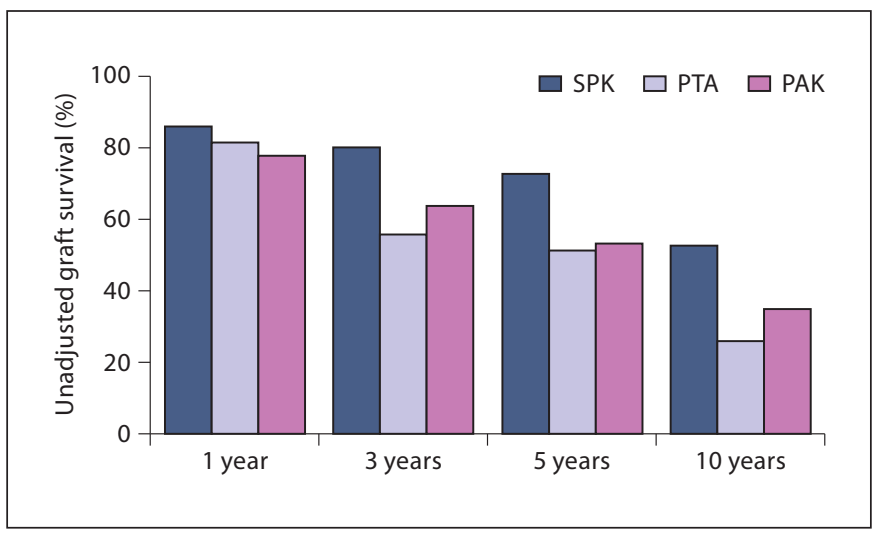

Fig. 1. Unadjusted 1-, 3-, 5- and 10-year pancreas graft survival by transplant type. Death is included as an event. From 2008 OPTN/ SRTR Annual Report, table 1.13.

Seven percent of pancreas transplants are performed as PTA which is generally reserved for patients in whom glucose control is extremely difficult to achieve and suffer from frequent episodes of life-threatening hypoglycemia [2]. Patients with a GFR of $<80 \mathrm{ml} / \mathrm{min}$ will be sensitive to the nephrotoxic immunosuppressive drugs and are likely to need a kidney transplant in the future [3].

PAK accounts for $17 \%$ of all pancreas transplants and involves transplantation of a pancreatic graft into a recipient with a well-functioning living or deceased donor kidney allograft. The benefits of PAK compared with SPK include the option of transplanting a kidney from a living donor [4]. The main disadvantage of PAK transplants is that the long-term pancreas graft survival rates remain lower than SPK (fig. 1). This difference is likely due to immunologic pancreas graft loss. It may be that rejection episodes are missed or diagnosed later because of the inability to use serum creatinine levels as a marker for rejection (since the kidney and pancreas grafts are from different donors). Some studies suggest that the immunologic problems that may have limited PAK transplantation may be ameliorated with newer immunosuppressive regimens leading to a reduction in the rejection rates [5].

\section{Timing of Pancreas Transplantation}

The demand is much higher in the US for deceased donor kidneys, and therefore it is the need for a deceased donor kidney in an SPK that determines the waiting time. The waiting time varies due to local allocation policies but is, for most patients, more than 1 year. The median waiting time for PAK transplants is significantly lower. The current recommendation is to do the pancreas transplant as soon as the recipient adequately recovers from the kidney transplant operation and has stable renal function. This is especially important for regions with a long waiting time for an SPK. At least $50 \%$ of candidates waiting for SPK die while waiting for more than 4 years [6]. Using living kidney donors also allows for a preemptive transplant (before the patient needs to be on dialysis).

While most patients will be on dialysis at the time of transplant, in $22 \%$ of all simultaneous transplants the kidneys are transplanted preemptively [2]. Recipients of a preemptive SPK have better survival 8 years after transplantation when compared with those transplanted while already on dialysis [7]. Registry data also suggest that preemptive SPK is associated with improved kidney allograft survival compared to non-preemptive SPK [8].

It is important to note that there is inconsistency in the literature regarding the relative benefit of SPK over living kidney alone transplant in patients with type 1 diabetes. This inconsistency reflects that it is difficult to fully control for baseline differences. Data suggest that SPK recipients with a functioning pancreas graft have superior survival compared with living kidney alone transplant [9]. Others found that SPK does not seem to grant a survival advantage compared with kidney transplant alone (KTA) from a living donor [10]. These conflicting data create challenges to the clinician in advising which option may be best for an individual patient with a potential living donor.

\section{Recipient Criteria}

The most common indication for pancreas transplantation is C-peptide-deficient type 1 diabetes mellitus. A small percentage of transplants, roughly $8 \%$, have been in recipients with type 2 diabetes [11]. Rarely, the procedure has been performed in cases of abnormal exocrine function [12]. The considerations in patient selection entail criteria specific to the pancreas transplant and common to all potential recipients. This patient population is at high risk of cardiovascular complications. This is evident in the fact that $30 \%$ of asymptomatic patients with type 1 diabetes mellitus with end-stage renal failure will have substantial coronary artery stenosis on angiography [13]. Therefore, uncorrected cardiovascular dis- 
ease is the most common contraindication to pancreas transplantation. $15 \%$ of all pancreas transplant recipients are older than 50 years of age, and while these patients have a higher risk of postoperative complications, age per se is not considered to be an absolute contraindication [14]. However, a thorough cardiac workup often including coronary angiography is critical in this patient population.

\section{Surgical Procedure}

There are two major technical decisions to be made during pancreatic graft implantation - how to manage exocrine secretions and how to manage venous outflow. The two options in managing the exocrine secretions of the pancreas are the use of bladder drainage or enteric drainage, the latter is preferred by most centers. Enteric drainage is performed as a side-to-side duodenojejunostomy at $30-60 \mathrm{~cm}$ from the ligament of Treitz or as a Roux-en-Y anastomosis and carries the complication of an enteric leak.

A major theoretical advantage of bladder drainage is that it facilitates the detection of pancreas rejection by measuring urinary amylase levels and avoids an enteric anastomosis. Disadvantages are that exocrine drainage of the pancreas graft in the bladder is associated with the loss of large quantities of bicarbonate-rich pancreatic secretions in the urine, leading to metabolic acidosis and stone formation [2]. These complications frequently require enteric conversion.

Most pancreatic allografts have been transplanted using the iliac vasculature for inflow and outflow. Advantages of this approach include lower rates of allograft thrombosis and the ability to use either the bladder or intestine for drainage of exocrine secretions. This approach, however, results in peripheral hyperinsulinemia with portal hypoinsulinemia, because the first-pass effect of hepatic degradation is lost. The actual clinical impact of this peripheral hyperinsulinemia remains uncertain. Portal drainage is more physiologic, but the real clinical impact remains unproven and its use is sporadic and center-specific [2]

\section{Complications}

Early postoperative complications include bleeding, infection, rejection, graft pancreatitis, duodenal leaks, and graft thrombosis. Early arterial or venous graft thrombosis can occur in as many as $5-10 \%$, and is the most common early cause of loss of a pancreatic allograft $[2,15]$. Liberal use of Doppler ultrasound imaging should be employed for any indication of early graft dysfunction.

Early duodenal segment leaks tend to result from technical complications or ischemia. Late duodenal leaks tend to be caused by rejection, infection, or ischemia of the duodenal staple line. Leaks do not result in an alteration of endocrine function, but present with elevated white blood cell counts, graft tenderness, fever, and can lead to a pancreaticocutaneous fistula or peripancreatic abscess [15].

Intra-abdominal infections are much more common after pancreas transplantation than after kidney transplantation and represent a significant cause of mortality if inadequately treated [15].

The overall incidence of posttransplant diabetes mellitus is $17 \%$. Factors associated with the development of persistent diabetes mellitus after pancreas transplantation include high pretransplant BMI, high pretransplant daily insulin requirement and the occurrence of acute rejection [16]. Patients with posttransplant diabetes mellitus have normal to elevated C-peptide levels which may be secondary to exogenous weight gain or to the effects of immunosuppressants. It is important to perform pancreatic graft biopsies to distinguish between rejection, recurrence of type 1 diabetes mellitus, and the occurrence of type 2 diabetes mellitus.

\section{Pancreas and Kidney Graft Survival}

The best pancreas allograft survival rates, $86 \%$ at one year and $53 \%$ at 10 years, are achieved with SPK transplants. One-year graft survival rates for PAK and PTA are 77 and $81 \%$, respectively, and 10 -year rates were 35 and $26 \%$, respectively (fig. 1). Of note, one-year and 5-year survivals for solitary grafts continue to improve and will hopefully translate into improved mortality.

The unadjusted kidney graft survival for SPK transplants is the highest in SPK patients with functioning pancreas and is $72 \%$ at 7 years after transplantation compared with $60 \%$ SPK with subsequent loss of pancreas graft function [9]. These data are in agreement with a retrospective cohort study that found that SPK transplants were associated with improved kidney allograft survival versus kidney alone transplants [8]. 


\section{Patient Survival}

Not unexpectedly, the patient survival is worse compared to waitlisted patients in the first 90 days after transplant, reflecting the stress of surgery; however, at 4 years after transplant, survival for SPK recipients far exceeds that of their waitlisted counterparts, and is no worse for PAK and PTA recipients. The most influential factors leading to patient mortality were shown to be graft failure and return to exogenous insulin [6].

The patient survival rate of pancreas transplant recipients improved over the last several years and is now more than $95 \%$ at 1 year [14]. Whether the benefits of pancreas transplantation translate into increased survival is controversial. Most recent Scientific Renal Transplant Registry data showed that patient survival rates were similar for PAK, SPK, and PTA through the first 3 posttransplant years, but the 5- and 10-year patient survival rates were lower for PAK recipients: $65 \%$ at 10 years compared with 70 and $73 \%$ for SPK and PTA recipients, respectively.

SPK recipients can expect to live 15 years longer than type 1 diabetics on the wait list who do not receive a transplant. In addition, SPK recipients can expect to live 10 years longer than if they were to receive a KTA [17]. Therefore, pancreas transplantation is justified on the basis of the mortality data but depends on recipient age and preservation of pancreas function [6].

\section{Impact of Pancreas Transplantation on Vascular Disease, Neuropathy, and Quality of Life}

Most data suggest that euglycemia can stop the progression of diabetic nephropathy, but these effects to show take up to 10 years. The benefits of euglycemia on kidney function might be underestimated as they are being counteracted by the nephrotoxic effects of the immunosuppressive drugs.

By 2 years after transplant, KTA recipients showed elevated creatinine and urine albumin levels compared with those in SPK recipients. Furthermore, biopsy data suggest that pancreas transplantation can prevent or reverse mild histologic changes of diabetic nephropathy $[18,19]$. As mentioned earlier, data also suggest that improved diabetes control has beneficial effects on kidney allografts as SPK is associated with improved kidney allograft survival versus kidney alone transplants $[8,9]$.

At least $75 \%$ of patients with type 1 diabetes develop retinopathy by 10 years, and a significant proportion will develop blindness [20]. Data suggest a regression of diabetic retinopathy in $43 \%$ of patients given SPK versus $23 \%$ given KTA. However, $50 \%$ of both groups showed no benefit, although follow-up was only one year [21]. The results of studies of diabetic retinopathy after pancreas transplantation are difficult to interpret, being confounded by small patient numbers, short follow-up and differing control groups. Its is clear that most benefits become apparent after few years [22]. Continued ophthalmic follow-up is necessary as some patients will still have progression of their disease.

In patients who underwent successful pancreas transplantation, neurologic evaluation demonstrated a general trend toward improvement in the motor and sensory nerve conduction studies at 1 year and in autonomic function at 5 years $[23,24]$.

Quality of life is increasingly used to measure the success of an intervention such as transplantation. Quality of life studies in pancreas transplantation show improved perception about diabetes-specific issues such as satisfaction with diet flexibility and health management. The patient is relieved to be free from strict dietary restrictions, frequent blood sugar monitoring and insulin therapy [25].

\section{Rejection and Immunosuppression}

Pancreas transplantation is associated with immunologic graft loss as a result of alloimmunity or autoimmune recurrence [26]. Even transplants between identical twins need immunosuppression to prevent autoimmune recurrence [27]. Pancreas rejection rates are about 5-25\%, depending on the combination of immunosuppression that is used [12]. Because of the high prevalence of early pancreas rejection and the difficulty in its diagnosis, induction therapy (most commonly thymoglobulin) is routinely included in immunosuppressive protocols for pancreas transplant recipients [12]. Most centers use tacrolimus over cyclosporine as the preferred calcineurin inhibitor combined with mycophenolate mofetil and steroids. Comparison of cyclosporine with tacrolimus in combination with mycophenolate mofetil, and steroid plus induction with antithymocyte globulin showed a reduction in the rates of severe rejection with much lower rates of pancreas graft loss at 3 years in the tacrolimus group [28].

To avoid insulin resistance, steroid-free regimen seems to be an attractive regimen in pancreas transplantation. An analysis of 200 consecutive SPK transplant 
patients who received tacrolimus and antibody induction and who were randomly assigned to either steroid therapy or avoidance, no significant differences were found in patient and graft survival and rejection rates [29].

Pancreas allografts are monitored by laboratory tests that assess the adequacy of the endocrine and exocrine functions, and by radiologic techniques that determine blood flow into the graft. The specificity of these markers in establishing the diagnosis of acute rejection is suboptimal. Clinical features for the diagnosis of acute rejection, such as elevated serum amylase or lipase levels, 50\% decrease in urinary amylase for bladder-drained grafts, unexplained fever, and hyperglycemia were associated with a positive predictive value of only $75 \%$. Hyperglycemia can result from multiple etiologies, and is frequently a sign of late or severe acute rejection. Pancreas allograft biopsy is the gold standard for the diagnosis of acute re- jection. Kidney biopsy, for easier accessibility, has been used as a surrogate for a pancreas biopsy in patients with an SPK transplant [28].

\section{Conclusion}

An SPK with both organs from the same deceased donor is the most common transplant involving the pancreas. Because of the worldwide shortage of deceased donors, living-kidney donation should be encouraged for all patients with end-stage renal disease and diabetes irrespective of whether they need a pancreas. The long-term advantages of this surgical procedure have to be balanced against the potential morbidity and mortality associated with it, and the side effects from the long-term immunosuppression that is needed to prevent alloimmunity and autoimmune recurrence.

\section{References}

1 Andreoni KA, Brayman KL, Guidinger MK, Sommers CM, Sung RS: Kidney and pancreas transplantation in the United States, 1996-2005. Am J Transplant 2007;7:13591375.

-2 Gruessner AC, Sutherland DE, Gruessner RW: Pancreas transplantation in the United States: a review. Curr Opin Organ Transplant 2010;15:93-101.

-3 Gruessner RW, Sutherland DE, Drangstveit MB, Kandaswamy R, Gruessner AC: Pancreas allotransplants in patients with a previous total pancreatectomy for chronic pancreatitis. J Am Coll Surg 2008;206:458-465.

$\checkmark 4$ Gruessner AC, Sutherland DE, Dunn DL, Najarian JS, Humar A, Kandaswamy R, Gruessner RW: Pancreas after kidney transplants in posturemic patients with type I diabetes mellitus. J Am Soc Nephrol 2001;12: 2490-2499.

-5 Larson TS, Bohorquez H, Rea DJ, Nyberg SL, Prieto M, Sterioff S, Textor SC, Schwab TR, Griffin MD, Gloor JM, Kudva YC, Kremers WK, Stegall MD: Pancreas-after-kidney transplantation: an increasingly attractive alternative to simultaneous pancreas-kidney transplantation. Transplantation 2004;77: 838-843.

6 Gruessner RW, Sutherland DE, Gruessner AC: Mortality assessment for pancreas transplants. Am J Transplant 2004;4:2018 2026.
7 Pruijm MT, de Fijter HJ, Doxiadis II, Vandenbroucke JP: Preemptive versus nonpreemptive simultaneous pancreas-kidney transplantation: a single-center, long-term, follow-up study. Transplantation 2006;81: 1119-1124.

8 Israni AK, Feldman HI, Propert KJ, Leonard $\mathrm{M}$, Mange KC: Impact of simultaneous kidney-pancreas transplant and timing of transplant on kidney allograft survival. Am J Transplant 2005;5:374-382.

-9 Weiss AS, Smits G, Wiseman AC: Twelvemonth pancreas graft function significantly influences survival following simultaneous pancreas-kidney transplantation. Clin J Am Soc Nephrol 2009;4:988-995.

10 Bunnapradist S, Cho YW, Cecka JM, Wilkinson A, Danovitch GM: Kidney allograft and patient survival in type I diabetic recipients of cadaveric kidney alone versus simultaneous pancreas kidney transplants: a multivariate analysis of the UNOS database. J Am Soc Nephrol 2003;14:208-213.

11 Nath DS, Gruessner AC, Kandaswamy R, Gruessner RW, Sutherland DE, Humar A: Outcomes of pancreas transplants for patients with type 2 diabetes mellitus. Clin Transplant 2005;19:792-797.

12 White SA, Shaw JA, Sutherland DE: Pancreas transplantation. Lancet 2009;373:1808-1817.

13 Ramanathan V, Goral S, Tanriover B, Feurer ID, Kazancioglu R, Shaffer D, Helderman $\mathrm{JH}$ : Screening asymptomatic diabetic patients for coronary artery disease prior to renal transplantation. Transplantation 2005; 79:1453-1458.
14 Gruessner AC, Sutherland DE: Pancreas transplant outcomes for United States (US) and non-US cases as reported to the United Network for Organ Sharing (UNOS) and the International Pancreas Transplant Registry (IPTR) as of June 2004. Clin Transplant 2005;19:433-455.

15 Sansalone CV, Maione G, Aseni P, Mangoni I, De Roberto A, Soldano S, Minetti E, Broggi ML, Civati G: Surgical complications are the main cause of pancreatic allograft loss in pancreas-kidney transplant recipients. Transplant Proc 2005;37:2651-2653.

16 Dean PG, Kudva YC, Larson TS, Kremers WK, Stegall MD: Posttransplant diabetes mellitus after pancreas transplantation. Am J Transplant 2008;8:175-182.

17 Ojo AO, Meier-Kriesche HU, Hanson JA, Leichtman A, Magee JC, Cibrik D, Wolfe RA, Port FK, Agodoa L, Kaufman DB, Kaplan B: The impact of simultaneous pancreas-kidney transplantation on long-term patient survival. Transplantation 2001;71:82-90.

18 Coppelli A, Giannarelli R, Vistoli F, Del Prato S, Rizzo G, Mosca F, Boggi U, Marchetti P: The beneficial effects of pancreas transplant alone on diabetic nephropathy. Diabetes Care 2005;28:1366-1370

19 Fioretto P, Steffes MW, Sutherland DE, Goetz FC, Mauer M: Reversal of lesions of diabetic nephropathy after pancreas transplantation. N Engl J Med 1998;339:69-75. 
20 Giannarelli R, Coppelli A, Sartini M, Aragona M, Boggi U, Vistoli F, Rizzo G, Del Prato S, Mosca F, Marchetti P: Effects of pancreaskidney transplantation on diabetic retinopathy. Transpl Int 2005;18:619-622.

-21 Wang Q, Klein R, Moss SE, Klein BE, Hoyer C, Burke K, Sollinger HW: The influence of combined kidney-pancreas transplantation on the progression of diabetic retinopathy. A case series. Ophthalmology 1994;101:10711076.

-22 Chow VC, Pai RP, Chapman JR, O’Connell PJ, Allen RD, Mitchell P, Nankivell BJ: Diabetic retinopathy after combined kidneypancreas transplantation. Clin Transplant 1999; 13:356-362.

-23 Kennedy WR, Navarro X, Goetz FC, Sutherland DE, Najarian JS: Effects of pancreatic transplantation on diabetic neuropathy. $\mathrm{N}$ Engl J Med 1990;322:1031-1037.
24 Martinenghi S, Comi G, Galardi G, Di Carlo V, Pozza G, Secchi A: Amelioration of nerve conduction velocity following simultaneous kidney/pancreas transplantation is due to the glycaemic control provided by the pancreas. Diabetologia 1997;40:1110-1112.

25 Sureshkumar KK, Patel BM, Markatos A, Nghiem DD, Marcus RJ: Quality of life after organ transplantation in type 1 diabetics with end-stage renal disease. Clin Transplant 2006;20:19-25.

26 Vendrame F, Pileggi A, Laughlin E, Allende G, Martin-Pagola A, Molano RD, Diamantopoulos S, Standifer N, Geubtner K, Falk BA, Ichii H, Takahashi H, Snowhite I, Chen Z, Mendez A, Chen L, Sageshima J, Ruiz P, Ciancio G, Ricordi C, Reijonen H, Nepom GT, Burke GW 3rd, Pugliese A: Recurrence of type 1 diabetes after simultaneous pancreas-kidney transplantation, despite immunosuppression, is associated with autoantibodies and pathogenic autoreactive CD4 T-cells. Diabetes 2010;59:947-957.

-27 Sutherland DE, Sibley R, Xu XZ, Michael A, Srikanta AM, Taub F, Najarian J, Goetz FC: Twin-to-twin pancreas transplantation: reversal and reenactment of the pathogenesis of type I diabetes. Trans Assoc Am Physicians 1984;97:80-87.
28 Malaise J, De Roover A, Squifflet JP, Land W, Neuhaus P, Pratschke J, Kahl A, Pascher A, Boas-Knoop S, Arbogast H, Hoffmann J, Illner WD, Seissler, Schlamp, Viebahn, Wunsch, Hajt, Klar E, Scharek W, Hopt, Pisarski P, Drognitz O, Thurow C, Dette K, Bechstein WO, Woeste G, Klempnauer J, Becker T, Luck, Neipp, Konigsrainer A, Steurer W, Margreiter R, Mark, Bonatti, Saudek F, Boucek P, Adamec M, Havrdova T, Koznarova $R$, Vanrenterghem Y, Pirenne J, Maes B, Kuypers D, Coosemans W, Evenepoel P, van Ophem D, Marcelis V, van V, Peeters, de H, de R, Fernandez-Cruz L, Ricart MJ, Nakache R, Morel P, Berney T, Demuylder S: Immunosuppression in pancreas transplantation: the Euro SPK trials and beyond. Acta Chir Belg 2008;108:673-678.

-29 Axelrod D, Leventhal JR, Gallon LG, Parker MA, Kaufman DB: Reduction of CMV disease with steroid-free immunosuppression in simultaneous pancreas-kidney transplant recipients. Am J Transplant 2005;5:14231429. 